\title{
How Hydrogen Admixture Changes Plasma Jet Characteristics in Spray Processes at Low Pressure
}

\author{
Georg Mauer ${ }^{1}$
}

Received: 1 October 2020 / Accepted: 15 November 2020 / Published online: 1 December 2020 (c) The Author(s) 2020

\begin{abstract}
In plasma spraying, hydrogen is widely used as a secondary working gas besides argon. In particular under low pressure, there are strong effects on the plasma jet characteristics even by small hydrogen percentages. Under such conditions, fundamental mechanisms like diffusion and recombination are affected while this is less relevant under atmospheric conditions. This was investigated for argon-hydrogen mixtures by optical emission spectroscopy (OES). The small electron densities under the investigated low pressure conditions implied specific difficulties in the application of several OES-based methods which are discussed in detail. Adding hydrogen to the plasma gas effected an increased plasma enthalpy. Moreover, the jet expanded radially as the reactive part of the thermal conductivity was enhanced by recombination of atomic hydrogen so that the shock waves were less reflected at the cold jet rims. In the jet cores, the lowest temperatures were found for the highest hydrogen admixture because the energy consumption due to the dissociation of molecular hydrogen outbalanced the increase of the plasma enthalpy. Variations in the radial temperature profiles were related to the jet structure and radial thermal conductivity. The local hydrogen-argon concentration ratios revealed an accumulation of hydrogen atoms at the jet rims. Clear indications were found, that higher hydrogen contents promoted the fast recombination of electrons and ions. However, it is assumed that the transport properties of the plasma were hardly affected by this, since the electron densities and thus the ionization degrees were generally small due to the low pressure conditions.
\end{abstract}

Keywords Thermal spraying · Low pressure · Hydrogen · Optical emission spectroscopy · Plasma diagnostics

\section{Introduction}

$\mathrm{Ar}-\mathrm{H}_{2}$ mixtures are widely used in experimental devices and industrial processes, such as for example in plasma spraying, because they provide to particles injected in the plasma jet, on the one hand, a sufficiently high momentum through the argon gas, and on the other hand, a high heat transfer through the hydrogen gas [1]. Furthermore,

Georg Mauer

g.mauer@fz-juelich.de

1 Forschungszentrum Jülich GmbH, IEK-1, Jülich, Germany 
hydrogen permits to increase the voltage and therefore the plasma power since the plasma arc in the torch adjusts itself to a size which minimizes the heat flux from the core to the cooled anode wall. This arc constriction is controlled mainly by the thermal conductivity of the plasma gas and thus by the amount of hydrogen admixture. As a consequence, the arc resistance, which is inversely proportional to the effective arc cross section, and thus the voltage and power are increased.

An essential characteristic of some novel plasma spray processes like very low pressure plasma spraying (VLPPS) and plasma spray-physical vapor deposition (PS-PVD) [2] is the small plasma density in the jet. Here, the low electron densities imply a disposition to thermodynamic non-equilibrium. Under such conditions, hydrogen affects fundamental mechanisms like diffusion and recombination while this is less relevant under atmospheric conditions at equilibrium.

In this work, optical emission spectroscopy (OES) was applied to study such phenomena in low pressure plasma jets. Analyses of the intensities and the broadening of emission lines were performed to determine electron densities and plasma temperatures. Furthermore, local variations of the plasma gas composition were investigated. Particular difficulties in applying some OES-based methods due to the low electron densities are discussed in detail.

\section{Experimental Methods}

\section{Plasma Spraying}

Experiments were carried out on an Oerlikon Metco Multicoat VLPPS/PS-PVD System (Oerlikon Metco, Wohlen, Switzerland) which resulted from a comprehensive reconstruction of an existing conventional LPPS system. In particular, the system is equipped with an additional vacuum pumping unit, a large vacuum blower to provide sufficient pumping capacity at low pressures, enlarged cooling capacity, additional power sources, and a new torch transfer system.

A single cathode F4-VB gun was used with a nozzle throat diameter of $7 \mathrm{~mm}$. The spray parameters are given in Table 1 . The plasma characterization by OES was conducted without any feedstock injection, nevertheless a carrier gas flow was fed through the two feedstock injectors.

Table 1 Spray parameters

\begin{tabular}{ll}
\hline Plasma gas $^{\mathrm{a}}$ & $\mathrm{Ar} 50 \mathrm{slpm}$ \\
& $\mathrm{H}_{2} \quad 0-12 \mathrm{slpm}$ \\
Chamber pressure & $150 \mathrm{~Pa}$ \\
Current & $600 \mathrm{~A}$ \\
Voltage & $35.6-74.8 \mathrm{~V}$ \\
Input power & $21-44.5 \mathrm{~kW}$ \\
Net power & $10.8-26 \mathrm{~kW}$ \\
Carrier gas $^{\mathrm{a}}$ & $\mathrm{Ar} 2 \times 2 \mathrm{slpm}$ \\
\hline
\end{tabular}

${ }^{\mathrm{a}}$ slpm standard liters per min 


\section{Optical Emission Spectroscopy}

The spectrometer applied for OES was the ARYELLE 200 (Laser Technik Berlin (LTB), Berlin, Germany) scanning a wavelength range of 381-786 $\mathrm{nm}$. Plasma radiation was detected through a borosilicate glass window in the process chamber. The collected radiation was transferred through an achromatic lens and an optical fiber to the $50 \mu \mathrm{m}$ entrance slit of the spectrometer, and then detected by a $1024 \times 1024$ CCD array. The system is equipped with an Echelle grating and the spectral resolution capability is 22,000 (17.3-35.7 pm). Calibration was carried out using a spectral $\mathrm{Hg}$ lamp.

Plasma characterization was performed by intensity and broadening analyses of specific emission lines. At low pressure conditions, broadening analysis is advantageous as it does not rely on specific thermodynamic equilibrium conditions, but only on Maxwell kinetic energy balance for atoms and electrons, respectively [3]. Different mechanisms contributing to emission line broadening can be distinguished [4]:

- Stark broadening is caused by collisions between charged particles with exited emitting atoms. For neutral atom lines, electron-impact broadening produces a symmetrical, shifted profile of the Lorentzian type. Ion-contribution introduces asymmetry.

- Doppler broadening is an effect of the thermal motion of excited atoms. The line intensity distribution is Gaussian.

- Instrumental broadening is introduced by the spectrometer and shows approximately Gaussian profiles. It must be determined by means of a laser source or spectral lamp to be able to subtract it from other broadening contributions.

- Van der Waals, natural, and resonance broadening are not relevant under the examined conditions.

The effects giving rise to Gaussian line shapes tend to be independent from those producing Lorentzian broadening profiles. As such, the convolution of a Gauss function with a Lorentz function results in the Voigt function [5] where the Gauss contribution determines the profile core while the Lorentzian part governs the far wings.

The selection of the spectral lines for diagnosis purposes has to be done carefully. The most widely used one, particularly for broadening analyses, is the $\mathrm{H}_{\beta}$ line of the hydrogen Balmer series at $486.135 \mathrm{~nm}$ because of its large Stark width [6]. It is relatively isolated, does not show significant self-absorption, and is almost independent on $T_{e}$ and ion dynamics effects [7]. The latter can be considerable if using other species like He I. The Stark profile of the $\mathrm{H}_{\alpha}$ line at $656.279 \mathrm{~nm}$ is closer to a Lorentz function but the broadening is considerably smaller. $\mathrm{The}_{\gamma} \mathrm{H}_{\gamma}$ line at $434.047 \mathrm{~nm}$ is relatively weak so that only their intensities were evaluated in this work and not their profile shapes. There are also some well investigated Ar I lines, e.g. at $696.543 \mathrm{~nm}$ [8]. However, non-hydrogenic lines are generally not suitable for broadening analyses at low pressure where $n_{e}<10^{15} \mathrm{~cm}^{-3}$ as their Stark width is too small. This is generally true also for their Doppler width. But, with the applied spectrometer, the $\mathrm{H}_{\beta}$ line is supported by sufficient measured data points. Thus, broadening analyses could be performed only for hydrogen containing plasmas while other evaluation methods relying just on the line intensities and not on the line shapes were applied in this work also using non-hydrogenic lines (Ar I). The areas under the Voigt fitting curves which are direct parameters of the Voigt function were considered to represent the measured peak intensities $I$. 
Figure 1 shows the evolution of $\mathrm{H}_{\beta}$ emission line at increasing hydrogen addition to the plasma. For a primary gas flow rate of $50 \mathrm{slpm} \mathrm{Ar,} \mathrm{a} \mathrm{minimum} \mathrm{addition} \mathrm{of} 2 \mathrm{slpm} \mathrm{H}_{2}$ was found as sufficient to obtain well-developed $\mathrm{H}_{\beta}$ peaks which could be fitted reliably as indicated by high coefficients of determination $R^{2}>0.99$.

\section{Deconvolution of Emission Line Profiles}

In order to deconvolute the measured profiles to the Gauss and Lorentz constituent functions, one supposed straightforward approach could be to fit the measured data points with a Voigt function using a form where the Gaussian and the Lorentzian full widths at half maximum (FWHM) $\Delta \lambda_{G}$ and $\Delta \lambda_{L}$ are direct parameters [9]. Several researchers however, experienced that the results were not satisfactory in particular for low electron densities. Such conditions were specified by Konjević [10] to prevail below $n_{e}=10^{16} \mathrm{~cm}^{-3}$ and $n_{e}=10^{15} \mathrm{~cm}^{-3}$ for the $\mathrm{H}_{\alpha}$ and $\mathrm{H}_{\beta}$ line, respectively. He synthesized $\mathrm{H}_{\beta}$ line profiles using tabulated data and fitted Voigt profiles to them. The parameters of the synthesis and the results of the fitting procedure showed significant differences regarding the share of $\Delta \lambda_{G}$ and $\Delta \lambda_{L}$ in the total FWHM. In the present work, similar difficulties were experienced. There are several reasons. As already mentioned above, the profiles are not ideally shaped as the Lorentzian part may exhibit deviations, asymmetries, and shifts especially at low pressure conditions [11-14]. The electron density can fall below the limit where the line splits in several components and a central dip appears [15, 16]. Indeed, for the first three lines of the Balmer series $\mathrm{H}_{\alpha}, \mathrm{H}_{\beta}$, and $\mathrm{H}_{\gamma}$, fine structure limits (FSL) are reached at $n_{e}=6 \cdot 10^{14} \mathrm{~cm}^{-3}, n_{e}=4 \cdot 10^{13} \mathrm{~cm}^{-3}$, and $n_{e}=10^{13} \mathrm{~cm}^{-3}$ according to [17]. They are in the range of the conditions investigated in this work. Thus, the maximum values and the peak shapes may be affected. Figure 2 shows examples for shape deviation, asymmetry and peak splitting of the first and second line of the Balmer series $\mathrm{H}_{\alpha}$ and $\mathrm{H}_{\beta}$ measured at $500 \mathrm{~mm}$ spray distance on axis $(r=0 \mathrm{~mm})$ at the highest examined hydrogen addition (12 slpm).

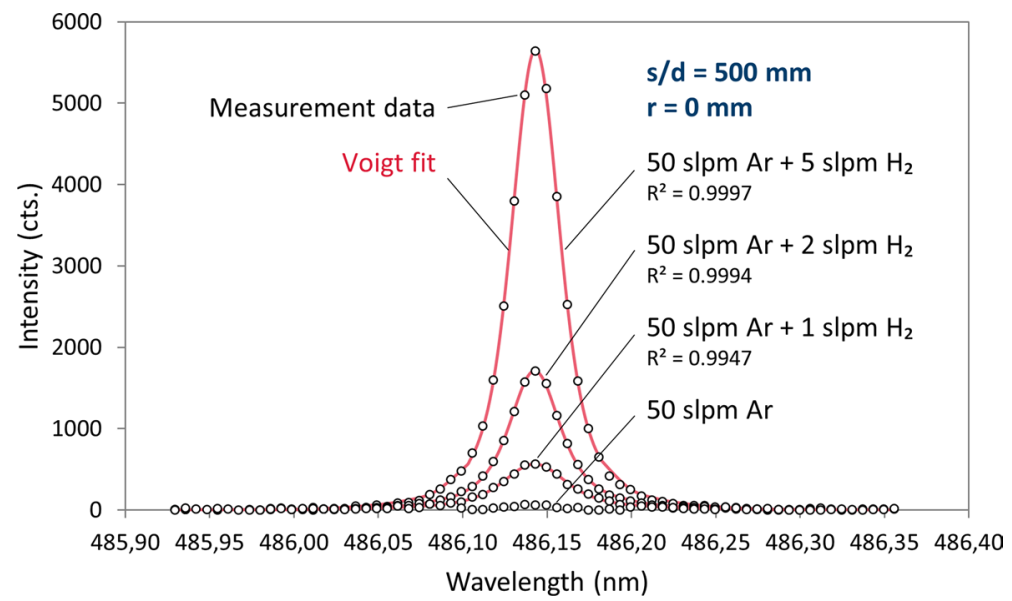

Fig. 1 Evolution of $\mathrm{H}_{\beta}$ emission line at increasing $\mathrm{H}_{2}$ addition to the plasma measured on axis $(\mathrm{r}=0 \mathrm{~mm})$ at $500 \mathrm{~mm}$ spray distance; the primary plasma gas flow rate was constantly $50 \mathrm{slpm}$ Ar; the data points were obtained from the spectrometric measurements, the solid lines signify the Voigt fits, $\mathrm{R}^{2}$ denotes the coefficients of determination 


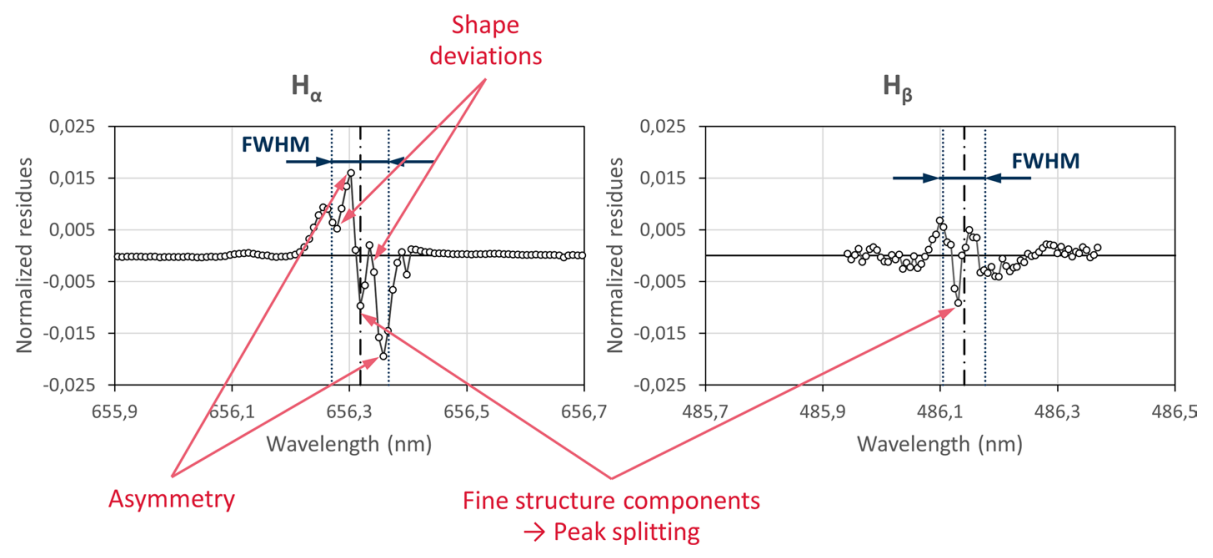

Fig. 2 Residues between spectroscopically measured intensities and Voigt fits normalized to the peak maxima for the first and second line of the Balmer series $H_{\alpha}$ and $H_{\beta}\left(12 \mathrm{slpm} \mathrm{H}_{2}+50 \mathrm{slpm} \mathrm{Ar,} \mathrm{s/d=500} \mathrm{mm,}\right.$ $\mathrm{r}=0 \mathrm{~mm})$

Likewise, at low electron densities, the ratio of $\Delta \lambda_{G}$ and $\Delta \lambda_{L}$ can get large so that the selectivity of the Voigt fitting process may be affected. This applies particularly to the $\mathrm{H}_{\alpha}$ line as the Stark width is smaller and the Doppler width is larger than for the $\mathrm{H}_{\beta}$ and $\mathrm{H}_{\gamma}$ lines.

Obviously, only small deviations of the measured profiles from ideal Voigt shapes affect the accurateness of the Voigt fits with regard to the deconvolution to their constituent functions while their total FWHMs, however, can be determined reliably. The application of alternative deconvolution methods in the frequency domain after Fourier transformation [18] did not solve the problem in this work (results not shown). Another workaround for the deconvolution problems is to apply a fixed Gaussian fraction [10]. For this, reasonable assumptions on the instrumental broadening $\Delta \lambda_{I}$ and the Doppler broadening $\Delta \lambda_{D}$ are required. The instrumental profile is assumed to have a Gaussian intensity distribution and was determined for the applied spectrometer by means of a low pressure mercury lamp by $\Delta \lambda_{I}=9.5 \mathrm{pm}$. If local thermodynamic equilibrium (LTE) is taken for granted, the heavy species' (ions and atoms) temperature $T_{h}$ is equal to the excitation temperature $T_{\text {exc }}$. Observing these equilibrium conditions carefully as shown below, $T_{e x c}$ was determined in this work by Boltzmann plots. Setting $T_{h}=T_{e x c}$, the Doppler width which is of Gaussian type as well can be obtained applying the well-known formula

$$
\Delta \lambda_{D}=2 \lambda_{0} \sqrt{\frac{2 k_{B} T_{h} \ln (2)}{M u c^{2}}}
$$

where $\lambda_{0}$ is the central wavelength of the emission line, $k_{B}$ is the Boltzmann constant, $M$ is the relative atomic mass of the absorbing atom, $u$ is the atomic mass unit, and $c$ is the speed of light. It is obvious that $T_{h} \propto \Delta \lambda_{D}^{2}$ so that the deconvolution problems mentioned above severely affect the accurateness of the determination of $T_{h}$. In fact, the analyses of Doppler half widths are known to require a high instrumental resolution [3].

The Gaussian fraction of the emission line's FWHM can now be calculated by

$$
\Delta \lambda_{G}=\sqrt{\Delta \lambda_{D}^{2}+\Delta \lambda_{I}^{2}}
$$


The total FWHM of the convoluted line profile $\Delta \lambda_{V}$ is determined by a Voigt fit of the measured data. The Lorentzian part $\Delta \lambda_{L}$ is complementary to the Gaussian fraction $\Delta \lambda_{G}$ and can be determined applying the approximate formula for convolution at half maximum given by Olivero and Longbothum [19] in a rearranged form

$$
\Delta \lambda_{L}=\Delta \lambda_{V}\left(7.7258-\sqrt{45.2358+14.4515 \frac{\Delta \lambda_{G}^{2}}{\Delta \lambda_{V}^{2}}}\right)
$$

Alternatively, simpler formulas can be applied [20, 21], however at some lower accuracy. $\Delta \lambda_{L}$ corresponds to the searched Stark width $\Delta \lambda_{S}$ as there is no other significant Lorentzian contribution to the peak broadening under the conditions examined in this work.

\section{Determination of Temperatures}

Methods to determine the electron temperatures $T_{e}$ which are based on Stark broadening $\Delta \lambda_{S}=\mathrm{f}\left(T_{e}, n_{e}\right)$ of two hydrogenic lines [22-24] are hardly applicable as the dependence of the $\mathrm{H}_{\alpha}, \mathrm{H}_{\beta}$, and the $\mathrm{H}_{\gamma}$ emission lines on $T_{e}$ is too weak below $n_{e}=10^{17} \mathrm{~cm}^{-3}$, see also Fig. 7 below. Consequently, methods based on intensity ratios of two emission lines [25], e.g., $\mathrm{H}_{\alpha} / \mathrm{H}_{\beta}$ or $\mathrm{H}_{\beta} / \mathrm{H} \gamma$, were not suitable as well. Another attractive method is based on the line to continuum intensity ratio [26-28]. But for the application of this method, the electron densities in the present work were again too low to produce sufficient continuum radiation.

In contrast, alternative methods rely on specific thermodynamic equilibrium conditions. Several diagnostic methods for instance, require complete thermodynamic equilibrium (CTE) [3] where kinetic, excitation, Saha, and radiation temperatures have very similar values. In contrast, improper balances consist of forward and backward processes not being each other's inverse [29]. Such improper balances are enhanced by ionization or recombination processes as there is an outward or inward net flow, respectively, of electrons. The weakest form of CTE departure is if part of the emitted radiation is not reabsorbed in the plasma and escapes, but Maxwell, Boltzmann and Saha balances still can be maintained. As spatial and time-related gradients are the reason, this is called local thermodynamic equilibrium (LTE) [29]. To maintain general LTE, a minimum electron density is required. E.g., assuming an electron temperature of $T_{e}=5000 \mathrm{~K}$, this critical electron density $n_{e, \text { crit }}$ can be estimated by the simple approach $n_{e, c r i t}=1.6 \cdot 10^{12} T_{e}^{1 / 2} \Delta E^{3} \mathrm{~cm}^{-3}$, where $\Delta E$ is the largest gap in the atomic (ionic) energy level system in $\mathrm{eV}$ [30]. For hydrogen, $1.2 \cdot 10^{17} \mathrm{~cm}^{-3}$ is obtained. As the results will show, $n_{e}$ is below under the examined conditions. Moreover, it should be noted that significant departures from LTE are generally observed in supersonic jets with steep gradients of plasma macroscopic properties due to the complex structure of the jet. The deviations from LTE are higher in the expansion than in the compression cells and very pronounced at the jet rims. Moreover, in coaction with very small characteristic flow times, kinetic non-equilibrium between electrons and heavy particles (atoms, ions) occurs [31] as the energy transfer between them is no longer sufficient to balance energy evenly. This leads to the two-temperature (2T) model [32] with the kinetic electron temperature $T_{e}$, the kinetic temperature of the heavy particles $T_{h}$, and the non-equilibrium parameter $\Theta=T_{e} / T_{h}$.

Nevertheless, provided that the kinetics of excitation and ionization processes are dominated by electron collisions, the population of excited atomic levels is Boltzmann distributed and ionization-recombination processes are in Saha equilibrium. Under such 
conditions which are referred to as electron excitation plasmas (EEK) [29], the excitation temperature $T_{e x c}$ can be regarded to represent the electron temperature $T_{e}$ and the ionization temperature $T_{\text {Saha }}$.

The expansion of the plasma into a low-pressure region is usually accompanied by rapid cooling and recombination. Under such conditions, if a recombination flow is dominated by collisional processes, lower energy levels are underpopulated with respect to Saha-Boltzmann equilibrium while high-lying states can be still in equilibrium with the ion ground state as these excited states are populated by the electron flux going from the recombination of ions and electrons to the ground state. The lower levels however, are depopulated by radiative decay [32]. This situation is referred to as partial local thermodynamic equilibrium (PLTE) [33]. Accordingly, in pure Ar plasmas with free electrons, only higher excited atomic states are found to be in Saha-Boltzmann equilibrium. As a consequence, the data points in the Boltzmann plots do not develop linearly [34]. Only those at higher energy levels represent $T_{e x c}, T_{\text {Saha }}$ and $T_{e}$ sufficiently. This situation is aggravated in $\mathrm{Ar}-\mathrm{H}_{2}$ mixtures where the presence already of small amounts of atomic hydrogen is found to significantly affect the plasma's state of equilibrium particularly at electron densities less than approx. $10^{15} \mathrm{~cm}^{-3}$. Here, atomic excited states are strongly underpopulated and meet the condition of Saha-Boltzmann equilibrium only very close to the ionization limit [35]. This can invalidate several common diagnostic methods [36]. For this reason, the emission lines which should be considered for Boltzmann plots must be chosen carefully as the emitting transitions must be in PLTE. Another important criterion for the selection of suitable emission lines is their possible tendency to self-adsorption. In principal, intense emitters are also strong absorbers. Regarding the spectrum of Ar I, this is true in particular for the emission lines above $750 \mathrm{~nm}$ indicating transitions from excited states on relatively low energy levels (e. g., $4 \mathrm{p}$ to $4 \mathrm{~s})$.

In Boltzmann plots, the atomic state distribution function (ASDF) is plotted against the energy level of the excited state $E_{k}$ for a set of emission lines of the same species and ionization state. The ASDF is calculated by

$$
A S D F=\ln \left(\frac{\varepsilon_{k i} \lambda_{k i}}{A_{k i} g_{k}}\right)
$$

where $\varepsilon_{k i}$ is the radiance of a spectral line emitted by the plasma due to the transition from an excited state $k$ to a lower energy state $i, A_{k i}$ is the transition probability, $g_{k}$ is the statistical weight (degeneracy) of the excited level $k$, and $\lambda_{k i}$ is the wavelength of emission. Figure 3 shows an example of a Boltzmann plot for neutral Ar measured on axis $(r=0 \mathrm{~mm})$ at standoff-distance $500 \mathrm{~mm}\left(5 \mathrm{slpm} \mathrm{H}_{2}+50 \mathrm{slpm} \mathrm{Ar}\right)$. For the linear regression, only transitions with high energy levels $E_{k}$ of the excited states were considered (transitions from $6 \mathrm{~s}$, $6 \mathrm{~d}, 5 \mathrm{~d}$, and $4 \mathrm{~d}$, respectively, to $4 \mathrm{p}$ ) to comply with PLTE. It is obvious that lower excited levels (transitions from $5 \mathrm{p}$ and $4 \mathrm{p}$, respectively, to $4 \mathrm{~s}$ ) are underpopulated and thus not in PLTE as their data points do not match with the straight line indicating the Boltzmann distribution. The mentioned energy levels can be seen in the Grotrian diagram in Fig. 4 with all transitions in the wavelength range covered by the used spectrometer 380-780 nm). The 15 selected emission lines and their data needed to calculate the ASDF are given in Table 2 .

With the slope $m$ of the regression line, the excitation temperature $T_{\text {exc }}$ can be calculated by 


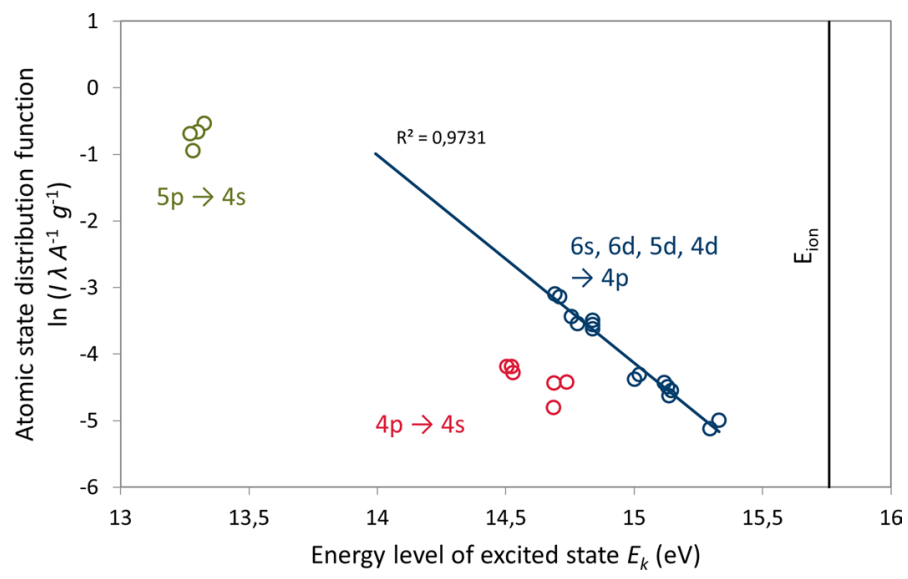

Fig. 3 Boltzmann plot for emission lines of neutral Ar with excited states close to the ionization energy; the slope of the straight regression line yields an excitation temperature of $3714 \pm 172 \mathrm{~K}\left(5 \mathrm{slpm} \mathrm{H}_{2}+50\right.$ slpm Ar, $\mathrm{s} / \mathrm{d}=500 \mathrm{~mm}, \mathrm{r}=0 \mathrm{~mm}$ )

Fig. 4 Grotrian diagram of neutral argon (radiatively linked levels in the wavelength range covered by the used spectrometer $380-780 \mathrm{~nm})$; redrawn from [37]

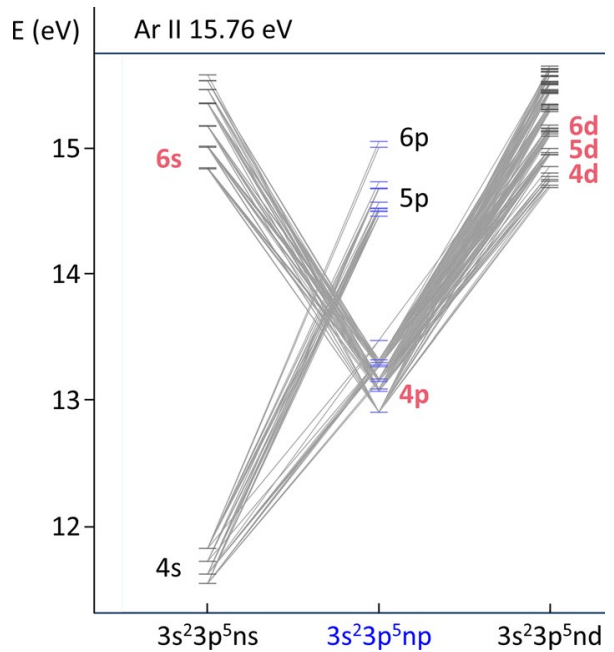

$$
T_{e x c}=\frac{-1}{m k_{B}}
$$

where $k_{B}$ is the Boltzmann constant $8.617 \cdot 10^{-5} \mathrm{eV} \mathrm{K}^{-1}$. The uncertainty of $T_{\text {exc }}$ can be estimated on the basis of the standard error of the slope.

At the larger applied stand-off distance of $800 \mathrm{~mm}$ and at off-axis positions $(\mathrm{r} \neq 0 \mathrm{~mm})$, only weak argon emission lines were found at the highest energy levels, in particular for the maximum hydrogen content of $12 \mathrm{slpm}$. Hence, Boltzmann plots for Ar I were not applicable anymore to determine the excitation temperatures in these cases. Instead, hydrogen emission lines were considered as an alternative. In the visible wavelength range, only the distinct lines of the Balmer series are available. As already mentioned above, only the first 
Table 2 Ar I emission lines which were considered for the determination of $\mathrm{T}_{\text {exc }}$ by Boltzmann plots; data taken from [37]

\begin{tabular}{lll}
\hline$\lambda_{k i}(\mathrm{~nm})$ & $E_{k}\left(\mathrm{~cm}^{-1}\right)$ & $A_{k i} \cdot \mathrm{g}_{\mathrm{k}} / \lambda_{k i}(\mathrm{~s} \cdot \mathrm{nm})^{-1}$ \\
\hline 518.7746 & 123,373 & $1.33 \cdot 10^{4}$ \\
549.5874 & 123,653 & $2.77 \cdot 10^{4}$ \\
555.8702 & 122,087 & $1.28 \cdot 10^{4}$ \\
560.6733 & 121,933 & $1.18 \cdot 10^{4}$ \\
591.2085 & 121,012 & $5.33 \cdot 10^{3}$ \\
603.2127 & 122,036 & $3.67 \cdot 10^{4}$ \\
604.3223 & 122,160 & $1.70 \cdot 10^{4}$ \\
641.6307 & 119,683 & $9.04 \cdot 10^{3}$ \\
687.1289 & 118,651 & $1.21 \cdot 10^{4}$ \\
693.7664 & 118,512 & $4.44 \cdot 10^{3}$ \\
703.0251 & 119,683 & $1.90 \cdot 10^{4}$ \\
720.6980 & 121,161 & $1.03 \cdot 10^{4}$ \\
735.3293 & 119,213 & $9.14 \cdot 10^{3}$ \\
737.2118 & 119,024 & $2.32 \cdot 10^{4}$ \\
743.5368 & 119,683 & $6.05 \cdot 10^{3}$ \\
\hline
\end{tabular}

three emission lines of this series $\mathrm{H}_{\alpha}, \mathrm{H}_{\beta}$, and $\mathrm{H}_{\gamma}$ were pronounced enough to be evaluable. They correspond to the transitions from principal quantum numbers (PQN) $3 \rightarrow 2,4 \rightarrow 2$, and $5 \rightarrow 2$, respectively. $\mathrm{H}_{\alpha}$ is the most powerful line. On the other hand, $\mathrm{H}_{\alpha}$ represents the transition which is most prone to non-equilibrium.

Several researchers gave criteria to assess the presence of PLTE for hydrogenic lines. Figure 5 gives corresponding critical electron densities for hydrogenic species as a function of the PQN of the exited state as specified by Griem [38] and van der Mullen [29] which are also in good agreement with [39]. The dependence on the electron temperature is weak or even not significant at all. Beyond the critical electron density, excited levels are considered to be collisional and thus not radiative, the latter means that they would be underpopulated with respect to the ground state.

As the results will show, the electron densities found in this work were sufficiently high to assume PLTE for hydrogen lines at PQN $\geq 4$. Hence, the $\mathrm{H}_{\alpha}$ line was not considered for Boltzmann plots, but the $\mathrm{H}_{\beta}$ and $\mathrm{H}_{\gamma}$ lines ( $\mathrm{PQN}=4$ and 5) could be utilized. Beyond, higher energetic lines $(\mathrm{PQN} \geq 6)$ were too weak in most of the examined cases. Hence, the Boltzmann plots contain only two data points so that the slope of the connecting straight line and thus the excitation temperature can be determined simply by calculating the gradient (line-pair method)

$$
m=\frac{1}{E_{k}^{(\beta)}-E_{k}^{(\gamma)}} \ln \left(\frac{\varepsilon_{k i}^{(\beta)} \lambda_{k i}^{(\beta)} A_{k i}^{(\gamma)} g_{k}^{(\gamma)}}{\varepsilon_{k i}^{(\gamma)} \lambda_{k i}^{(\gamma)} A_{k i}^{(\beta)} g_{k}^{(\beta)}}\right)
$$

where the superscripts $(\beta)$ and $(\gamma)$ denote the $\mathrm{H}_{\beta}$ and $\mathrm{H}_{\gamma}$ emission line, respectively. Since only two data points were involved, there was no degree of freedom to estimate the standard error of the slope.

To validate the excitation temperatures obtained on the basis of the $\mathrm{H}_{\beta}$ and $\mathrm{H}_{\gamma}$ lines, they were compared with $T_{e x c}$ determined on the basis of neutral Ar as exemplified in Fig. 3. Figure 6 shows this evaluation for measurements on axis $(r=0 \mathrm{~mm})$ where evaluable Ar lines were detectable. Only in the case of Ar at s/d=800 mm most of the 


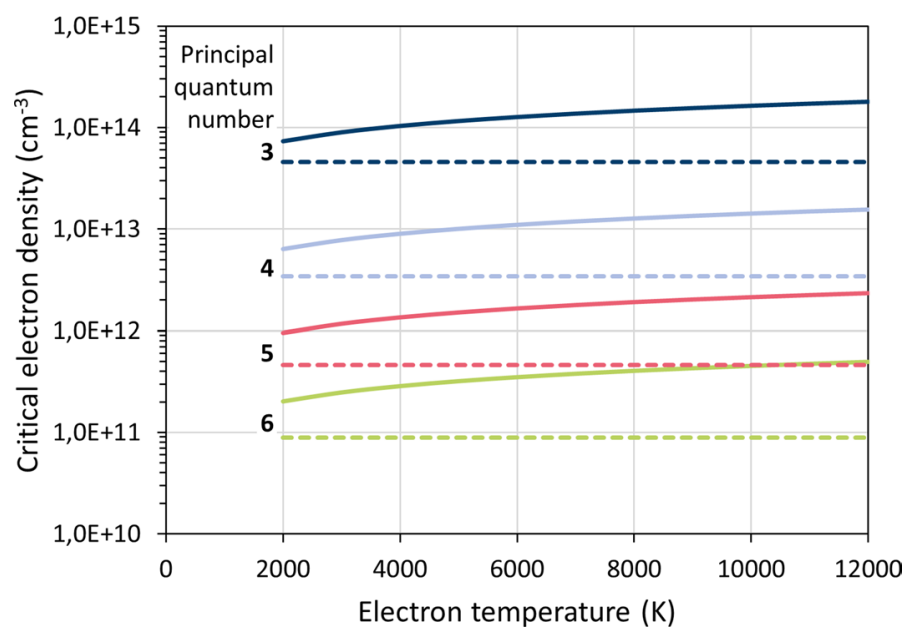

Fig. 5 Critical electron densities for the assumption of PLTE for hydrogenic species as a function of the PQN of the exited level as specified by Griem [38], Eq. 8 (solid lines), and van der Mullen [29], Eq. 7.22b (dashed lines)

applied emission lines were too weak. The excitation temperatures found on the basis of Ar and $\mathrm{H}$ show the same trends. However, those obtained on the basis of hydrogen are continuously lower than in the cases of Ar. Because it was not possible to calculate error bars for the hydrogen-based temperatures (as explained above) it is hard to assess whether these differences are due to a systematic error or due the nature of $\mathrm{Ar}-\mathrm{H}$ plasmas and potential non-equilibrium effects under low pressure conditions. Therefore, a merely practical approach was chosen: hereinafter, the hydrogen-based excitation temperatures $T_{e x c}$ were considered to represent the electron temperatures $T_{e}$ as these results were available even off-axis. Moreover, they were considered to represent the heavy species temperatures $T_{h}$ (atoms and ions) likewise as long as local thermodynamic equilibrium (LTE) could be taken for granted. The same precondition was made already for the deconvolution of the emission line profiles to their constituent functions (cp. " Deconvolution of Emission Line Profiles" section).

\section{Determination of Electron Densities}

Only few data are available in the literature on Stark broadening for very low electron densities $n_{e}<10^{16} \mathrm{~cm}^{-3}$. In this work, tabulated Stark $\mathrm{H}_{\beta}$ line broadening data published by Stehlé [40] was used, see Fig. 7, which was fitted in the range from $2500 \mathrm{~K}$ to $10,000 \mathrm{~K}$. In the following fitting formula, the very weak dependence on the electron temperature $T_{e}$ was averaged,

$$
\log n_{e}=1.301 \log \Delta \lambda_{S}+15.894
$$

where $n_{e}$ is electron density $\left(\mathrm{cm}^{-3}\right)$ and $\Delta \lambda_{S}$ is the Stark broadening (nm). Cross checks applying other semi-empirical formulas [7, 41-44] yielded similar results. 


\section{(a) Ar I}

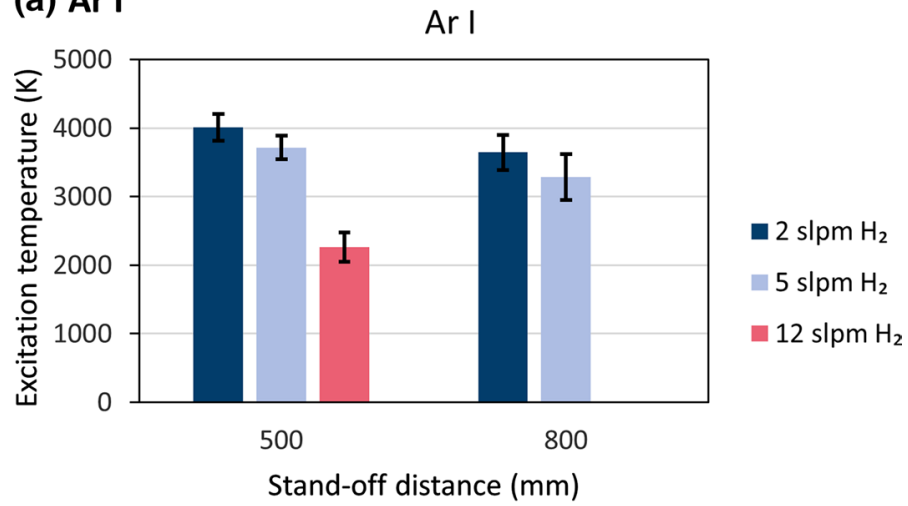

(b) $\mathrm{H}$

$\mathrm{H}$

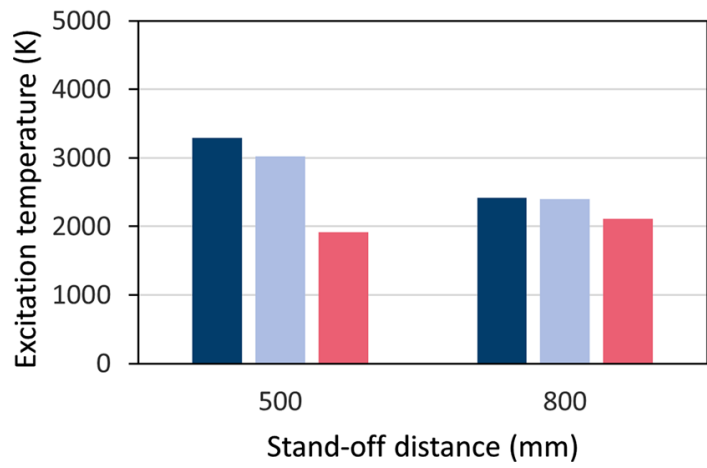

$\square 2$ slpm $\mathrm{H}_{2}$

5 slpm $\mathrm{H}_{2}$

12 slpm $\mathrm{H}_{2}$

Fig. 6 Excitation temperatures determined on axis $(\mathrm{r}=0 \mathrm{~mm})$ from Boltzmann plots of emission line intensities of (a) neutral Ar and (b) hydrogen in $500 \mathrm{~mm}$ and $800 \mathrm{~mm}$ stand-off distance, respectively; the case of $\mathrm{Ar}$ at $12 \mathrm{slpm} \mathrm{H}_{2}$ and $\mathrm{s} / \mathrm{d}=800 \mathrm{~mm}$ was not evaluable, as most of the lines were too weak; for Ar the error bars represent the uncertainties due to the standard error in the slope of the straight regression line in the Boltzmann plots; for hydrogen, uncertainties cannot be given since the linear is defined by two line intensities only $\left(\mathrm{H}_{\beta}\right.$ and $\left.\mathrm{H}_{\gamma}\right)$

\section{Determination of Constituent Concentrations}

In [45], a calibration-free method is presented to determine the species' concentrations and pressures based on the intercepts of the straight regression lines with the ordinate in Boltzmann plots in combination with electron densities obtained from Stark broadening. For this approach, at least one measurable emission line of each ionized species is required in addition to the lines of the neutrals. In this work however, no evaluable Ar II line could be detected under the examined conditions. As the results will show later, the degrees of ionization are very low so that the emission lines of the corresponding ions are obscured by the background radiation. And in the case of hydrogen, the ion is just a non-radiating proton. Hence, only single neutral emission lines were used in this work, to determine the ratio of the neutral constituents $R_{H \text {, Ar I }}$.

If the radiating transitions are Boltzmann distributed, the total density of a plasma species is proportional to 


$$
n_{t o t} \propto \frac{\varepsilon_{k i} Z(T) \lambda_{k i}}{g_{k} A_{k i}} \exp \left(E_{j} / k_{B} T_{e x c}\right)
$$

where $Z(T)$ is the partition function (sum over states). Hence, the concentration ratio of the two plasma constituents $\mathrm{H}$ and $\mathrm{Ar} \mathrm{I}$ is obtained by

$$
R_{H, A r I}=n_{t o t}^{(H)} / n_{t o t}^{(A r I)}
$$

To evaluate hydrogen-argon concentration ratios, the $\mathrm{H}_{\beta}$ and $\mathrm{Ar} \mathrm{I}(696.543 \mathrm{~nm})$ line intensities were chosen in this work. This Ar line has relatively low self-absorption [8]. An alternative would be Ar I at $738.398 \mathrm{~nm}$, but this is stronger and thus tends more to self-adsorption. Regarding the Balmer series of hydrogen, the strong $\mathrm{H}_{\alpha}$ is also prone to self-absorption. In consequence, the $\mathrm{H}_{\beta}$ is the best choice. The signal to noise ratio was good and the line shape less affected by effects like redistribution of radiation around the center [7]. For $T_{e x c}<10,000 \mathrm{~K}$, the partition functions of Ar I and $\mathrm{H}$ are equal to 1.0 and 2.0 , respectively.

\section{Abel Inversion}

To determine space-resolved radial distributions of the radiances $\varepsilon(r)$ in the axisymmetric plasma jet, Abel's transformation can be applied provided that the plasma is optically thin [46]. Values of $\varepsilon(r)$ (in units of power per unit volume and unit solid angle) can be obtained from the distribution of measured side-on intensities $I(y)$ (in units of power per unit area projected perpendicular to $x$ and unit solid angle), where $x$ is the measurement direction and $y$ is the vertical coordinate of the measurement position with respect to the horizontal jet axis $z$. The measured side-on intensities $I(y)$ are actually radiances laterally integrated over the $x$ coordinate through the jet. According to Abel, the radial distribution of the radiances $\varepsilon(r)$ can be obtained by solving the equation

$$
\varepsilon(r)=-\frac{1}{\pi} \int_{r}^{R} \frac{\frac{d I(y)}{d y}}{\sqrt{y^{2}-r^{2}}} d y
$$

where $R$ is the boundary radius of the radiating plasma jet.

The distribution of the side-on intensity $I(y)$ is usually obtained as a series of discrete measured values $I\left(y_{i}\right)$. They can be fitted by a symmetrical function $f(y)$. Based on this, two ways of solving Abel's equation were tried using the polynomial $f(y)=\sum_{n} a_{n} y^{n}, n=0(2) 8$. On the one hand, it was used as regression function to replace $I(y)$. For this, the derivative $\mathrm{d} f(y) / \mathrm{d} y$ can be given explicitly so that the integral in Abel's equation could be calculated exactly for any values of the radial coordinate $r$. On the other hand, this regression polynomial was used as an interpolation function so that the tables of Bockasten [47] with transformation coefficients for 20 data points could be applied to obtain radiances $\varepsilon(r)$ for the same number of discrete radial coordinates $r_{i}$.

It was found in both cases that $\varepsilon(r) \cdot R$ developed very similar to $I(y)$. Only close the jet boundary at $r=R$, both approaches yielded unstable results in some cases. Figure 8 shows the measured intensities $I\left(y_{i}\right)$, regression function $\mathrm{f}(y)$ and radial distribution of the radiances $\varepsilon(r) \cdot R$ obtained by Abel transformation for the $\mathrm{H}_{\alpha}$ line profile (example $\mathrm{H}_{2}$ admixture $12 \mathrm{slpm}$, spray distance $500 \mathrm{~mm}$ ). Thus, for the determination of electron temperatures and concentration ratios of plasma constituents as described in "Determination of 


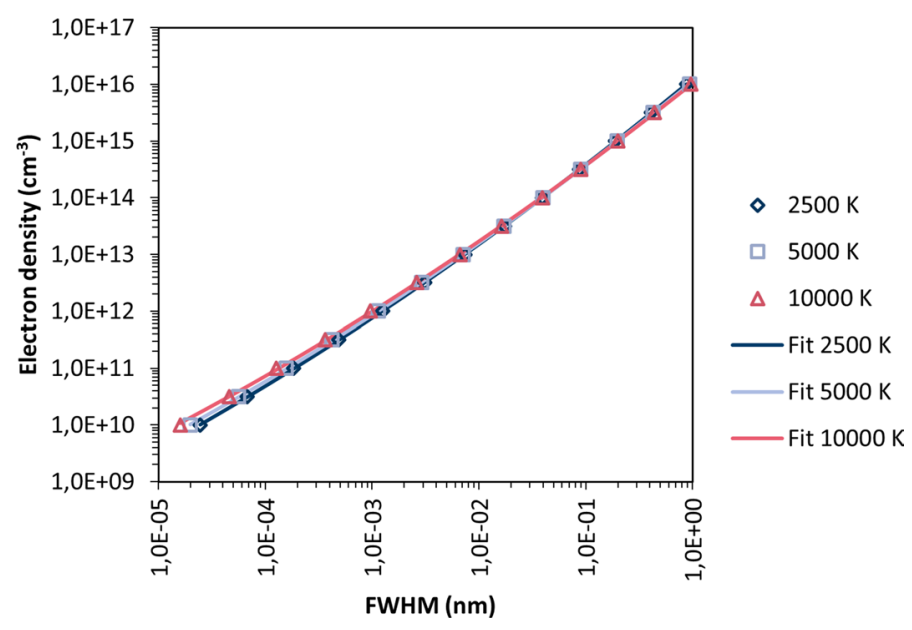

Fig. 7 Dependence of the Stark broadening (full width at half maximum) of the $\mathrm{H}_{\beta}$ line on electron density and electron temperature (fits of tabulated data published by Stehlé [40])

Temperatures" and "Determination of Constituent Concentrations" sections, respectively, Abel inversion was obsolete since only ratios of radial radiances $\varepsilon(r)$ are used. Thus, setting $r=y$, the side-on measured ratios of $I(y)$ could be used directly instead of $\varepsilon(r)$.

The same was found not only for the emission line intensities but also for the emission line spectral profiles. The discrete intensities $I\left(\lambda_{i}, y\right)$ of the $\mathrm{H}_{\alpha}$ line profile (same example $\mathrm{H}_{2}$ admixture $12 \mathrm{slpm}$, spray distance $500 \mathrm{~mm}$ ) measured at the wavelengths $\lambda_{i}$ (specified by the resolution of the spectrometer) were Abel-transformed to obtain the corresponding radial distribution of the radiance $\varepsilon\left(\lambda_{i}, r\right)$. These data points were fitted in the wavelength domain by a Voigt function as it was done for the measured intensities $I\left(\lambda_{i}, y\right)$. Hence, the spectral profile of the radiance $\varepsilon(\lambda, r)$ was obtained. Both of the specific spectral profiles $I(\lambda, y=0 \mathrm{~mm})$ and $\varepsilon(\lambda, r=0 \mathrm{~mm})$ showed almost the same FWHM of $50.5 \mathrm{pm}$ and $48.6 \mathrm{pm}$, respectively. Obviously, the emission line profiles showed identical shapes as the deviation was less than $4 \%$ and thus within the accuracy which can be expected due to the limited resolution in the wavelength domain. This is because the FWHM is invariant to the absolute line intensity. Hence, no Abel inversion was required for the determination of electron densities based on line broadening analyses as described in "Determination of Electron Densities" section.

\section{Results and Discussion}

\section{Effect of Hydrogen on the Plasma Enthalpy}

Due to the intense cooling of plasma torches, a cold boundary layer is formed in the plasma gas flow near to the anode wall. The remaining net part of the input power which is not dissipated increases the plasma gas enthalpy. Steenbeck's minimum principle [48] postulates a minimum arc voltage for a given arc current, working gas flow rate, and torch configuration. Although, this is a controversial issue [49], the fact is that the arc adjusts to a certain radial and longitudinal size. This leads also to the well-known restrike phenomenon [50]. 
The degree of the arc constriction is mainly controlled by the thermal conductivity of the plasma gas mixture at the flow temperature near the anode wall [51]. In contrast to argon, hydrogen has a relative high thermal conductivity below $6000 \mathrm{~K}$, due to the molecular dissociation.

Figure 9 (top) shows the effect of the hydrogen plasma gas content on the torch voltage and electrical input power using the F4-VB torch. Since the arc resistance is inversely proportional to the effective arc cross section, the arc voltage and correspondingly the electric input power increase as the current was kept constant. Figure 9 (bottom) shows the effect on the torch net power and torch efficiency. The net power was determined from the cooling water flow rate and the temperature difference between feed and return flow. Similar to the input power, the net power and thus the plasma enthalpy increases with the hydrogen content. The torch efficiency (ratio of net and input power) is almost not affected.

\section{Effect of Hydrogen on the Plasma Jet Structure}

Figure 10 shows the effect of hydrogen on the plasma jet structure under low pressure conditions (chamber pressure $150 \mathrm{~Pa}$ ). As mentioned above, the plasma net power is higher in case of hydrogen admixture. The jets are supersonic, i.e. pressure waves cannot travel upstream so that the conditions for arc generation in the torch are not affected by the expanding jets. Furthermore, they are under-expanded as the chamber pressure is lower than the plasma pressure at the nozzle exit. Here, the first expansion zone is formed followed by a strong drop to subsonic flow revealing the presence of a Mach reflection. The flow is accelerated further and a periodic structure of compression/expansion cells is observed until the local static pressure in the jet is in equilibrium with the surrounding pressure [52]. In case of hydrogen addition, the jet is widened radially as the reactive part of the thermal conductivity is enhanced by recombination of atomic hydrogen. Moreover, the shock waves are less reflected at the cold jet rims so that for pure Ar there are several subsequent expansion and compression cells, going even down to $800 \mathrm{~mm}$ jet length, while with hydrogen only one expansion and one compression cell could be identified.

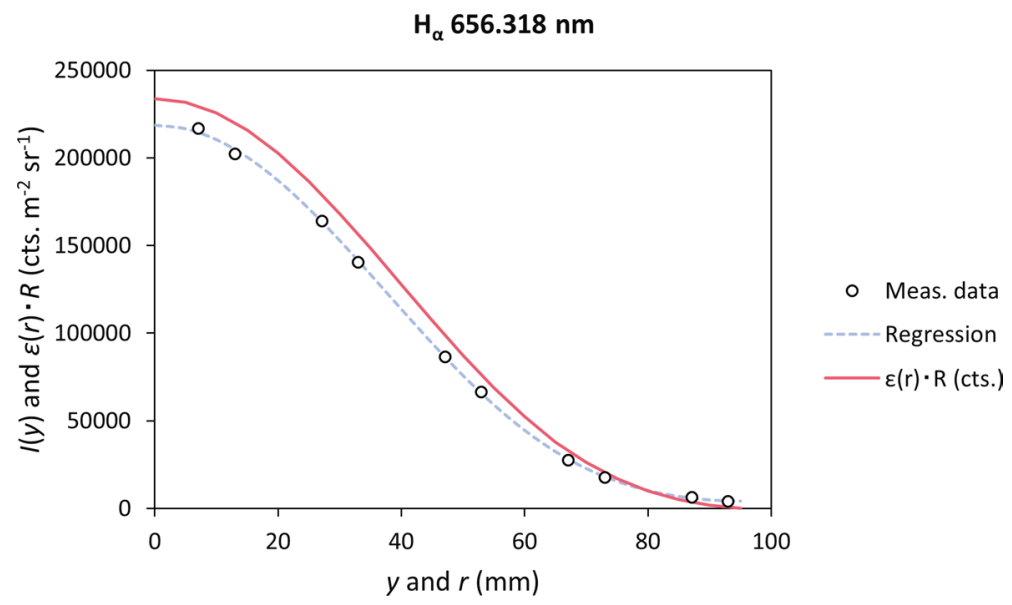

Fig. 8 Measured intensities $\mathrm{I}(\mathrm{y})$, regression function $\mathrm{f}(\mathrm{y})$ and radial distribution of the radiances $\varepsilon(\mathrm{r}) \cdot \mathrm{R}$ obtained by Abel transformation for the $\mathrm{H}_{\alpha}$ line profile (example $\mathrm{H}_{2}$ admixture 12 slpm, spray distance $500 \mathrm{~mm})$ 
Fig. 9 Effect of the hydrogen plasma gas content on the torch voltage and electrical input power (top) as well as on the torch net power and efficiency (bottom) using a F4-VB torch
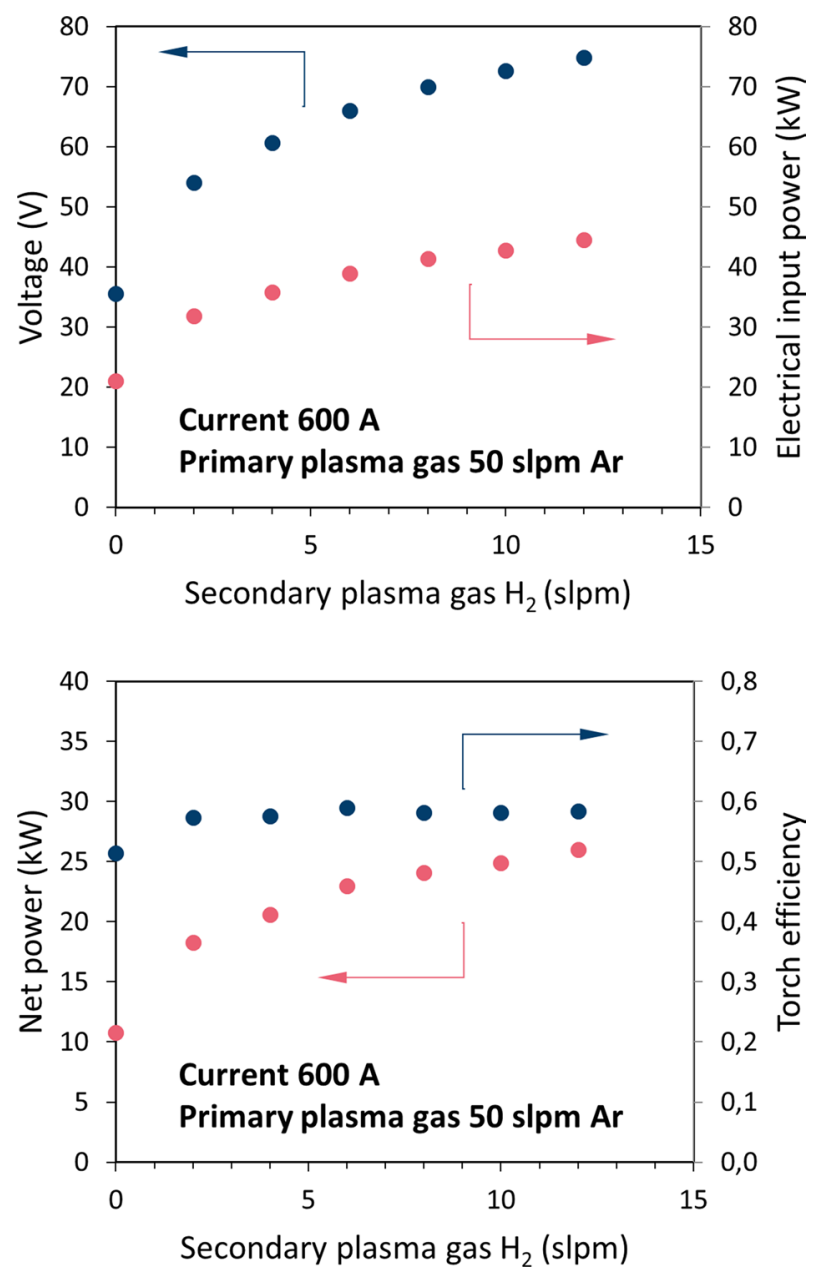

\section{Effect of Hydrogen on Plasma Characteristics}

\section{Temperatures}

Figure 11 shows the radial profiles of the excitation temperature $T_{e x c}$ at spray distances of $500 \mathrm{~mm}$ and $800 \mathrm{~mm}$ for three different hydrogen admixtures to the plasma gas. The areas under the Voigt fits were used to represent the intensities of the emission lines $\mathrm{H}_{\beta}$ and $\mathrm{H}_{\gamma}$. At the jet rims and at greater jet length these areas are relatively small with respect to the limited resolution of the spectrometer. Hence, their uncertainties increase und thus the uncertainties of $T_{\text {exc }}$ as expressed by the error bars.

As explained in "Determination of Temperatures" section, the excitation temperatures were considered to represent the electron temperatures $T_{e}$ and the heavy species temperatures $T_{h}$ (atoms and ions) as long as local thermodynamic equilibrium (LTE) could be taken for granted. The high values of $T_{e x c}$ at the jet rims are clear indications of non-equilibrium conditions, i.e. $T_{h} \neq T_{e x c}$ while $T_{e} \approx T_{e x c}$ may still hold. This suggestion is supported 
Fig. 10 Effect of hydrogen on the plasma jet structure under low pressure conditions; the photos were taken through the chamber window and the brightness was converted into rainbow pseudocolors (Color figure online)
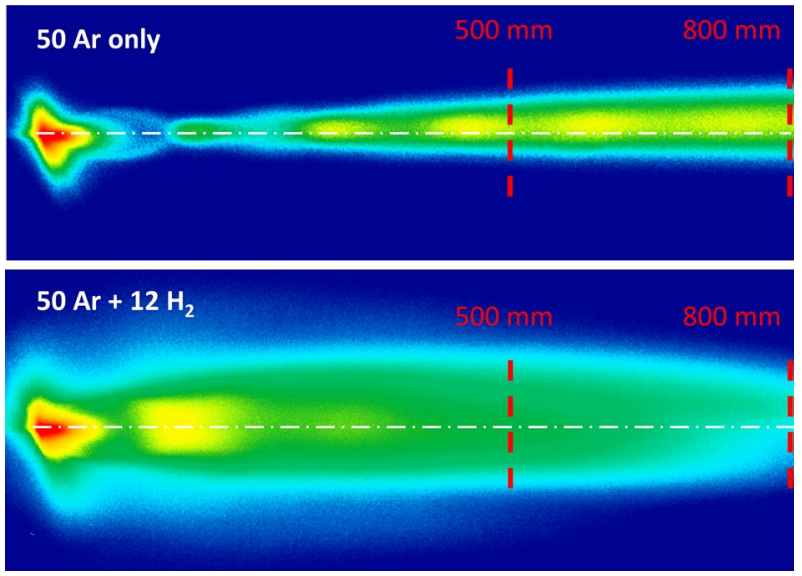

by the low electron densities at the jet rim dropping below the critical threshold for LTE, as shown in the next "Electron Densities" section. For the highest hydrogen content, $T_{\text {exc }}$ was lowest although the plasma enthalpy was the largest. But on the other hand, more energy was consumed for the dissociation of molecular hydrogen. Calculations of the plasma gas composition at chemical equilibrium [53, 54] (results are not shown here) at the present jet core temperatures (approx. 2100 to $2900 \mathrm{~K}$ ) and at chamber pressure (150 Pa) show, that the major hydrogen fraction is atomic. Hence, it is suggested that the hydrogen in the core of the expanded jets is still dissociated and the dissociation energy not released.

The radial temperature profiles broaden with increasing spray distance (s/d) as the jet diverges. At $500 \mathrm{~mm}$ spray distance, the broadening is most pronounced for the highest hydrogen content while at $800 \mathrm{~mm}$ the temperature profiles show similar shapes. The phenomena at $500 \mathrm{~mm}$ can be related to:

- Typical off-axis maxima in the radial profiles of expansion cells corresponding to barrel shock waves which are more pronounced for low hydrogen contents and pure Ar [31], see also Fig. 10;

- Higher reactional thermal conductivity in radial direction in the presence of hydrogen due to recombination.

\section{Electron Densities}

Figure 12 gives the radial profiles of the electron densities at spray distances of $500 \mathrm{~mm}$ and $800 \mathrm{~mm}$ for three different hydrogen admixtures to the plasma gas. They are of typical magnitude for expanding plasmas at low pressure $[31,55]$ and suggest low ionization degrees of just a few percent. Electron densities were not determined where non-equilibrium must be assumed at the jet rims, as explained before.

The radial profiles exhibit their maxima in the jet core and broaden with increasing spray distance (s/d) as the jets diverge. There are two effects on the electron densities. On the one hand, the plasma power raises with increasing hydrogen content leading to higher plasma enthalpy and degrees of ionization. On the other hand, higher hydrogen contents promote the recombination of electrons and ions, as will be explained below. At $500 \mathrm{~mm}$ spray distance, these effects are more or less balanced; but at $800 \mathrm{~mm}$, recombination dominates so that the 


\section{$\mathrm{s} / \mathrm{d}=500 \mathrm{~mm}$}

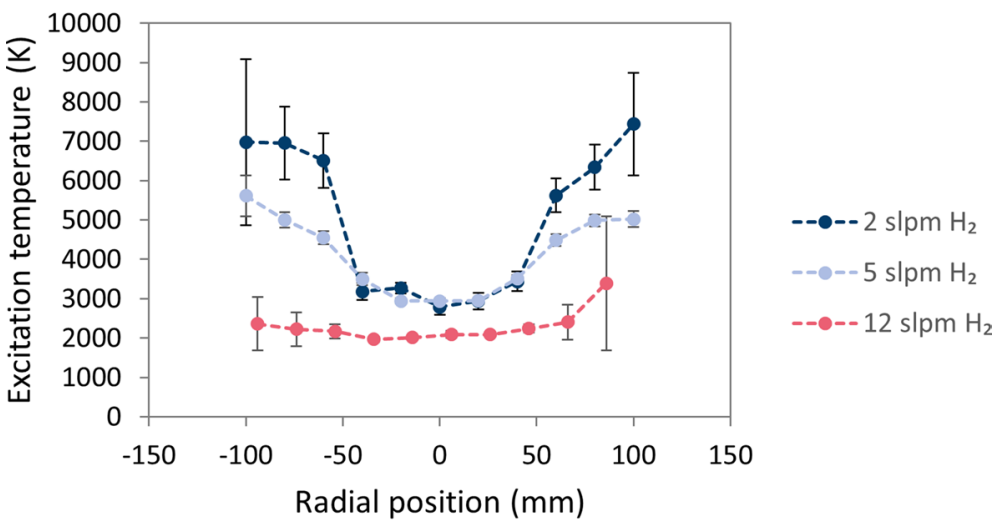

$\mathrm{s} / \mathrm{d}=800 \mathrm{~mm}$

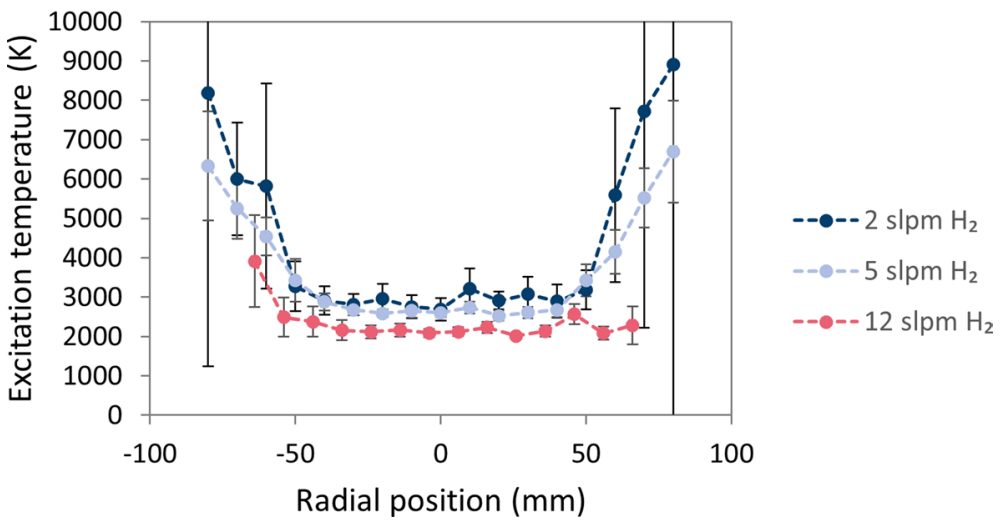

Fig. 11 Radial profiles of the excitation temperature at spray distances of $500 \mathrm{~mm}$ and $800 \mathrm{~mm}$ for three different hydrogen admixtures to the plasma gas; the error bars represent the uncertainties of the areas under the Voigt fits which were used to represent the emission line intensities

electron densities are lowered if the hydrogen content is increased. These effects could affect the transport properties of the plasma, namely the reactive part of the thermal conductivity. However, regarding the present low ionization degrees, such effect is assumed to be small.

In [35] it is reported that pure argon jets appeared to be chemically frozen. In contrast, argon-hydrogen jets under low pressure conditions were found to be less frozen. With increasing hydrogen content, they became strongly recombining. On the basis of experimental [56] and modeling [57] results it is suggested that soon after onset of jet expansion an associative charge exchange of $\mathrm{Ar}^{+}$and $\mathrm{H}_{2}$ occurs

$$
\mathrm{Ar}^{+}+\mathrm{H}_{2} \rightarrow \mathrm{ArH} \mathrm{H}^{+}+\mathrm{H}
$$

where $\mathrm{H}_{2}$ is created by catalytical recombination of $\mathrm{H}$ radicals on dust particles and the chamber surface, re-entering the plasma jet. This is followed by the dissociative recombination of the formed $\mathrm{ArH}^{+}$ 


$$
A r H^{+}+e^{-} \rightarrow A r+H^{*}
$$

Consequently, a strong decrease of $\mathrm{Ar}^{*}$ line intensities and dominant $\mathrm{H}^{*}$ lines are observed (* indicates the excited states). This is consistent with the emission spectra measured in this work as can be seen in Fig. 13. Moreover, while $\mathrm{Ar}^{+}$emission lines were hardly detectable here, in other experiments using a more powerful $03 \mathrm{CP}$ torch (results are not shown here), besides $\mathrm{Ar}^{*}$, also the $\mathrm{Ar}^{+}$line intensities decreased significantly when hydrogen was added to the plasma gas. This confirms the proposed recombination route. It is very effective so that already small hydrogen additions of a few percentages can decrease the ion density by three or four orders of magnitude [58]. The corresponding reaction rates of $k=1.1 \times 10^{-15} \mathrm{~m}^{3} \mathrm{~s}^{-1}$ and $k \approx 10^{-13} \mathrm{~m}^{3} \mathrm{~s}^{-1}$ respectively, are virtually independent on the electron temperature $T_{e}[56,59]$ and thus faster than three-particle recombination in pure $\mathrm{Ar}$

\section{$\mathrm{s} / \mathrm{d}=\mathbf{5 0 0} \mathrm{mm}$}

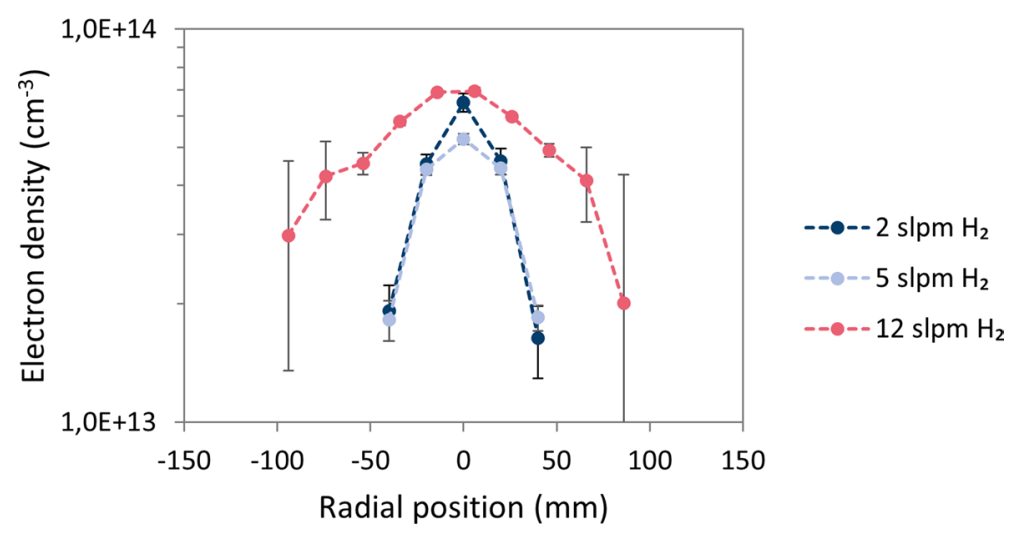

\section{$\mathrm{s} / \mathrm{d}=\mathbf{8 0 0} \mathrm{mm}$}

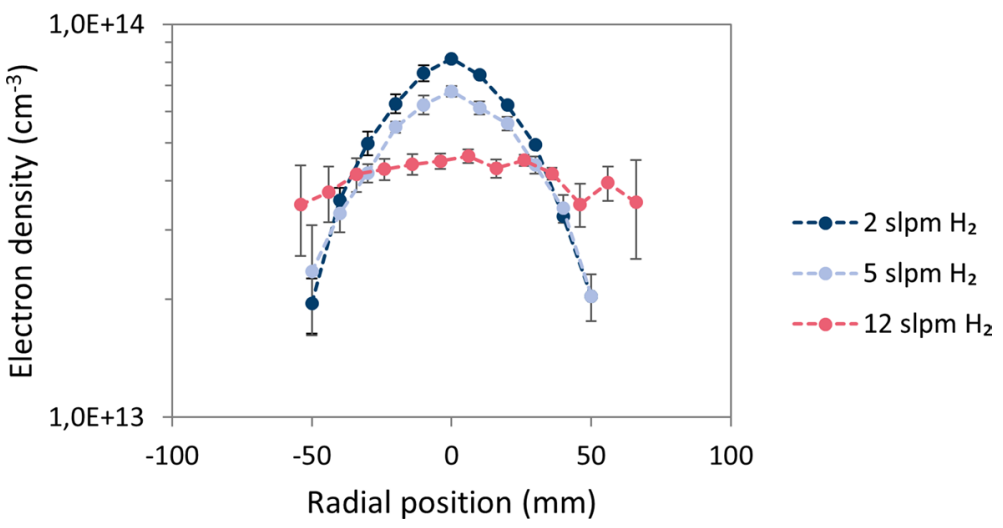

Fig. 12 Radial profiles of the electron densities at spray distances of $500 \mathrm{~mm}$ and $800 \mathrm{~mm}$ for three different hydrogen admixtures to the plasma gas; the error bars represent the uncertainties of the emission line intensities and the temperatures 


$$
A r^{+}+2 e^{-} \rightarrow A r^{*}+e^{-}
$$

with a reaction rate $k \propto T_{e}^{-9 / 2}$ (two-particle recombination $\mathrm{Ar}^{+}+e^{-} \rightarrow A r$ can be neglected) [60]. The reaction of $\mathrm{Ar}^{+}$and $\mathrm{H}_{2}$ to form $\mathrm{Ar}$ and $\mathrm{H}_{2}{ }^{+}$followed by a dissociative recombination $[56,58]$ is also of little importance for energies below $0.5 \mathrm{eV}$. Another reaction involving vibrationally excited hydrogen molecules reacting with $\mathrm{H}^{+}[56,58]$ appears unlikely as only low $\mathrm{H}^{+}$concentrations were observed [61].

\section{Concentration Ratios of Constituents}

Figure 14 shows the radial profiles of the hydrogen-argon concentration ratios $n_{H} / n_{A r I}$ at spray distances of $500 \mathrm{~mm}$ and $800 \mathrm{~mm}$ for three different hydrogen admixtures to the plasma gas. It is obvious that the highest concentration ratios are found for the largest amount of hydrogen admixture of $12 \mathrm{slpm}$. It is difficult to relate these ratios to the H/Ar input ratios fed to the torch as the extent of excitation is different between $\mathrm{H}$ and Ar due to the reactions discussed above in the "Electron Densities" section. An accumulation of $\mathrm{H}$ atoms at the jet rims is observed in all cases; at the shorter spray distance of $500 \mathrm{~mm}$ it is more pronounced than at $800 \mathrm{~mm}$. The decrease of the profile wings indicate, that the concentration of hydrogen in the chamber far away from the jet is low.

Obviously, in the expansion of a plasma generated from an $\mathrm{Ar}-\mathrm{H}_{2}$ mixture, hydrogen atoms are decoupled from argon atoms. On the one hand, a mass focusing effect (segregation effect) is reported for supersonic expansions so that the heavier species could be concentrated in the center of the jet (cp. atomic weights $m_{H}=1 \mathrm{u}, m_{A r}=39.95 \mathrm{u}$ ) $[62,63]$. On the other hand, Mazouffre et al. [64] observed that for hydrogen, there is no discontinuity (or jump) in the axial density profile through the stationary shock front in the supersonic expansion, which is in contrast to Ar. Due to the corresponding non-conservation of forward flux of the atomic hydrogen, $\mathrm{H}$ radicals are supposed to radially escape the core of the jet. One order of magnitude radial loss of $\mathrm{H}$ flux was found which could not be explained just by the mass focusing effect. In consequence, $\mathrm{H}$ atoms are poorly confined within the supersonic domain of the plasma jet as the Ar shock structure is partially transparent for $\mathrm{H}$. Furthermore, due to the considerable recombination of $\mathrm{H}$ at the vessel walls and thus the (quasi)absence of atomic $\mathrm{H}$ in the background atmosphere, a significant gradient of the hydrogen radical density all over the expansion is assumed driving the radial outward diffusion [65].

$\operatorname{Ar} 1$

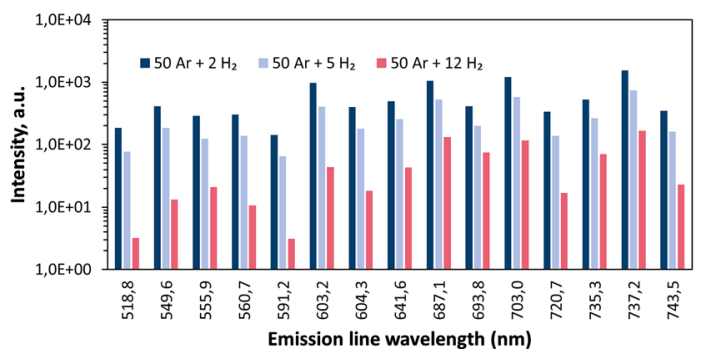

H

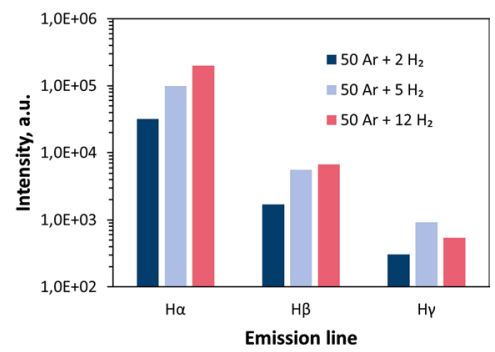

Fig. 13 Emission line intensities for neutral argon (left) and hydrogen (right) as a function of the amount of hydrogen admixture measured on axis $(\mathrm{r}=0 \mathrm{~mm})$ at spray distance $500 \mathrm{~mm}$ 


\section{$\mathrm{s} / \mathrm{d}=\mathbf{5 0 0} \mathrm{mm}$}

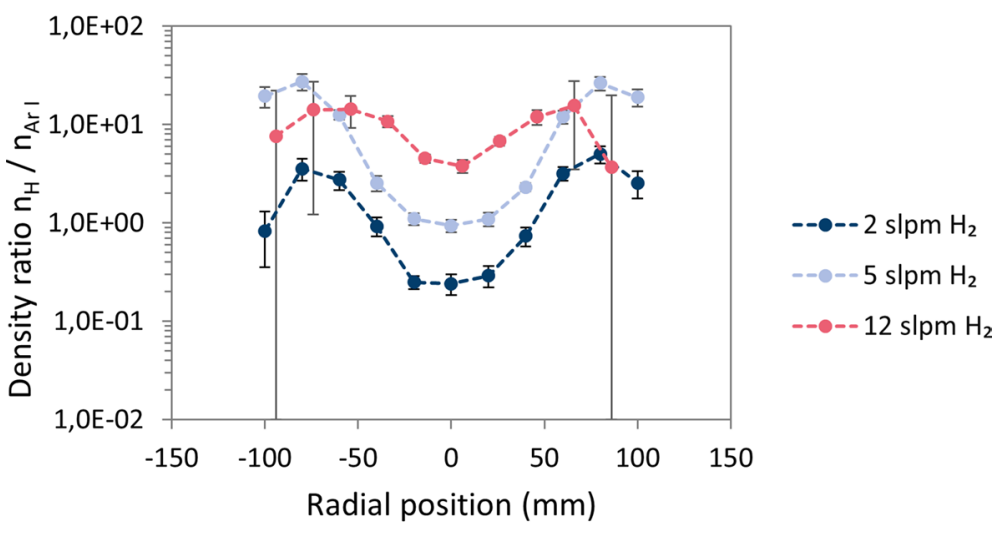

$\mathrm{s} / \mathrm{d}=\mathbf{8 0 0} \mathrm{mm}$

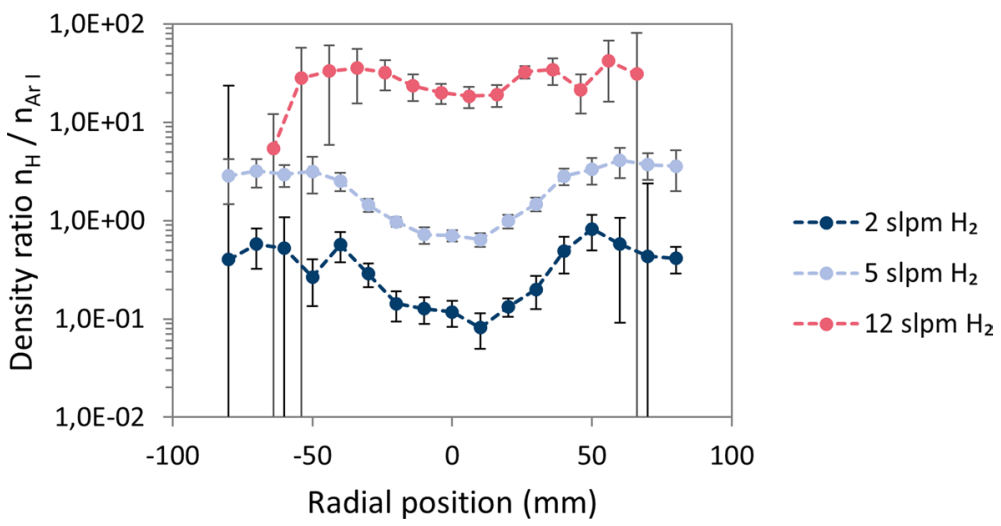

Fig. 14 Radial profiles of the hydrogen-argon concentration ratios at spray distances of $500 \mathrm{~mm}$ and $800 \mathrm{~mm}$ for three different hydrogen additions to the plasma gas; the error bars represent the uncertainties of the emission line intensities and the temperatures

\section{Conclusions}

Low pressures generally imply small electron densities and ionization degrees. Under such conditions, the application of OES-based methods and the selection of species and emission lines for evaluation must be done carefully with respect to the required equilibrium conditions and detectability of spectral features. In this work, the investigation of argon-hydrogen plasma gas mixtures at low pressure showed that already small hydrogen contents can change the plasma jet characteristics considerably.

In the core of the plasma jets, PLTE could be assumed. Hence, the excitation temperatures determined here were used to represent also the electron and heavy species temperature. In contrast, high values of $T_{e x c}$ at the jet rims were clear indications of nonequilibrium conditions. Although the plasma enthalpy was increased by the admixture of hydrogen, more energy was consumed for the dissociation of the hydrogen molecules. Thus, the lowest core temperatures were found in case of the highest hydrogen content. 
Differences in the radial temperature profiles were related to the jet structure and radial thermal conductivity.

The electron densities were affected due to the presence of hydrogen since a fast recombination route emerged for ions and electrons. This led also to a shift of the excited radiating species from argon to hydrogen. In principle, the reactive part of the plasma thermal conductivity could be enhanced by such recombination reactions. In this work however, the effect is obviously low since the ionization degrees are small under the examined low pressure conditions.

The local composition of the expanding plasma jet varied considerably. Obviously, hydrogen atoms could segregate from argon atoms and escape rapidly from the jet in radial direction.

These findings can contribute to an improved understanding of the prevailing mechanisms in VLPPS and PS-PVD plasma jets and to the development of advanced simulation models, particularly for low pressure conditions cp. e.g., [66].

Acknowledgments The authors gratefully acknowledge Dr. Wenting He and MSc. Cong Zhao, who carried out the OES measurements during their time at IEK-1 in Jülich, Hiltrud Moitroux, IEK-1, who took the photos, as well as fruitful discussions with Prof. Armelle Vardelle, Prof. Gilles Mariaux and Dr. Dmitrii Ivchenko, University of Limoges, IRCER.

Funding Open Access funding enabled and organized by Projekt DEAL.

Open Access This article is licensed under a Creative Commons Attribution 4.0 International License, which permits use, sharing, adaptation, distribution and reproduction in any medium or format, as long as you give appropriate credit to the original author(s) and the source, provide a link to the Creative Commons licence, and indicate if changes were made. The images or other third party material in this article are included in the article's Creative Commons licence, unless indicated otherwise in a credit line to the material. If material is not included in the article's Creative Commons licence and your intended use is not permitted by statutory regulation or exceeds the permitted use, you will need to obtain permission directly from the copyright holder. To view a copy of this licence, visit http://creativecommons.org/licenses/by/4.0/.

\section{References}

1. Rat V, Andre P, Aubreton J, Elchinger MF, Fauchais P, Lefort A (2002) Two-temperature transport coefficients in argon-hydrogen plasmas-I: elastic processes and collision integrals. Plasma Chem Plasma Process 22(4):453-474

2. Mauer G, Gindrat M, Smith MF (2016) Very Low Pressure Plasma Spraying (VLPPS), including PS-TF, PS-PVD, and PS-CVD. In: A. Vardelle et al. The 2016 Thermal Spray Roadmap. J Therm Spray Technol 25 (8):1383-1388. https://doi.org/10.1007/s11666-016-0473-x

3. Wiese WL (1991) Spectroscopic diagnostics of low temperature plasmas: techniques and required data. Spectrochim Acta B 46(6):831-841. https://doi.org/10.1016/0584-8547(91)80084-G

4. Griem HR (1997) Principles of plasma spectroscopy, vol 2. Cambridge monographs on plasma physics. University Press, Cambridge

5. Voigt W (1912) Über das Gesetz der Intensitätsverteilung innerhalb der Linien eines Gasspektrums. Sitzungsber Math-Phys Kl Bayer Akad Wiss Muenchen 25(III):603-620

6. Konjević N, Ivković M, Sakan N (2012) Hydrogen Balmer lines for low electron number density plasma diagnostics. Spectrochim Acta B 76:16-26. https://doi.org/10.1016/j.sab.2012.06.026

7. Gigosos MA, González MÁ, Cardeñoso V (2003) Computer simulated Balmer-alpha, -beta and -gamma Stark line profiles for non-equilibrium plasmas diagnostics. Spectrochim Acta B 58(8):14891504. https://doi.org/10.1016/s0584-8547(03)00097-1

8. Haddad GN, Farmer AJD (1984) Temperature determinations in a free-burning arc: 1. Experimental techniques and results in argon. J Phys D Appl Phys 14:1189-1196

9. Mauer G, Vaßen R (2012) Plasma spray-PVD: plasma characteristics and impact on coating properties. J Phys Conf Ser 406:012005. https://doi.org/10.1088/1742-6596/406/1/012005 
10. Konjević N, Ivkovic M, Sakan N (2012) Hydrogen Balmer lines for low electron number density plasma diagnostics. Spectrochim Acta B 76:16. https://doi.org/10.1016/j.sab.2012.06.026

11. Djurović S, Nikolić D, Savić I, Sörge S, Demura AV (2005) Asymmetry of $\mathrm{H}_{\beta}$ Stark profiles in T-tube hydrogen plasma. Phys Rev E 71(3):036407. https://doi.org/10.1103/PhysRevE.71.036407

12. Swafford LD, Surmick DM, Witte MJ, Woods AC, Gautam G, Parigger CG (2014) Hydrogen Balmer series measurements in laser-induced air plasma. J Phys Conf Ser 548:012049. https://doi. org/10.1088/1742-6596/548/1/012049

13. Halenka J, Olchawa W, Madej J, Grabowski B (2015) Pressure shift and gravitational redshift of Balmer lines in white dwarffs: rediscussion. Astrophys J 808(2):131. https://doi. org/10.1088/0004-637x/808/2/131

14. Grabowski B, Madej J, Halenka J (1987) The impact of the pressure shift of hydrogen lines on "relativistic" masses of white dwarfs. Astrophys J 313:750

15. Ehrich H, Kelleher DE (1980) Experimental investigation of plasma-broadened hydrogen Balmer lines at low electron densities. Phys Rev A 21(1):319-334. https://doi.org/10.1103/PhysRevA.21.319

16. Ivković M, Konjević N, Pavlović Z (2015) Hydrogen Balmer beta: the separation between line peaks for plasma electron density diagnostics and self-absorption test. J Quant Spectrosc Radiat Transf 154:1-8. https://doi.org/10.1016/j.jqsrt.2014.11.014

17. Olchawa W, Olchawa R, Grabowski B (2004) Stark broadening of hydrogen spectral lines with fine structure effects. Eur Phys J D 28(1):119-124. https://doi.org/10.1140/epjd/e2003-00291-5

18. Vogman GV, Shumlak U (2011) Deconvolution of Stark broadened spectra for multi-point density measurements in a flow Z-pinch. Rev Sci Instrum 82(10):103504. https://doi.org/10.1063/1.3647975

19. Olivero JJ, Longbothum RL (1977) Empirical fits to the Voigt line width: a brief review. J Quant Spectrosc Radiat Transf 17(2):233-236. https://doi.org/10.1016/0022-4073(77)90161-3

20. van de Hulst HC, Reesinck JJM (1947) Line breadths and voigt profiles. Astrophys J 106:121. https:// doi.org/10.1086/144944

21. Váczi T (2014) A new, simple approximation for the deconvolution of instrumental broadening in spectroscopic band profiles. Appl Spectrosc 68(11):1274-1278. https://doi.org/10.1366/13-07275

22. Torres J, Jonkers J, van de Sande MJ, van der Mullen JJAM, Gamero A, Sola A (2003) An easy way to determine simultaneously the electron density and temperature in high-pressure plasmas by using Stark broadening. J Phys D Appl Phys 36:L55-L59

23. Torres J, van de Sande MJ, van der Mullen JJAM, Gamero A, Sola A (2006) Stark broadening for simultaneous diagnostics of the electron density and temperature in atmospheric microwave discharges. Spectrochim Acta B 61(1):58-68. https://doi.org/10.1016/j.sab.2005.11.002

24. Becker de Moos B, Kessler B, Seelig W (1993) Determination of the electron density in an argon laser plasma by spectroscopy of the hydrogen $\mathrm{H}_{\alpha}$ and $\mathrm{H}_{\beta}$ lines. Contrib Plasma Phys 33(4):275-284

25. Siepa S, Danko S, Tsankov TV, Mussenbrock T, Czarnetzki U (2014) On the OES line-ratio technique in argon and argon-containing plasmas. J Phys D Appl Phys 47(44). https://doi.org/10.1088/00223727/47/44/445201

26. Bastiaans GJ, Mangold RA (1985) The calculation of electron density and temperature in Ar spectroscopic plasmas from continuum and line spectra. Spectrochim Acta B 40(7):885-892. https://doi. org/10.1016/0584-8547(85)80059-8

27. Sola A, Calzada MD, Gamero A (1995) On the use of the line-to-continuum intensity ratio for determining the electron temperature in a high-pressure argon surface-microwave discharge. J Phys D Appl Phys 28(6):1099-1110. https://doi.org/10.1088/0022-3727/28/6/012

28. Moon H-Y, Smith BW, Omenetto N (2012) Temporal behavior of line-to-continuum ratios and ion fractions as a means of assessing thermodynamic equilibrium in laser-induced breakdown spectroscopy. Chem Phys 398:221-227. https://doi.org/10.1016/j.chemphys.2011.07.002

29. van der Mullen JAM (1990) Excitation equilibria in plasmas; a classification. Phys Rep 191(2):109220. https://doi.org/10.1016/0370-1573(90)90152-R

30. Huddlestone RH (1965) Plasma diagnostic techniques, vol 21. Academic Press, New York, NY

31. Selezneva SE, Sember V, Gravelle DV, Boulos MI (2002) Spectroscopic validation of the supersonic plasma jet model. J Phys D Appl Phys 35:1338-1349

32. Calzada MD, Moisan M, Gamero A, Sola A (1996) Experimental investigation and characterization of the departure from local thermodynamic equilibrium along a surface-wave-sustained discharge at atmospheric pressure. J Appl Phys 80(1):46-55. https://doi.org/10.1063/1.362748

33. Rat V, Murphy AB, Aubreton J, Elchinger MF, Fauchais P (2008) Treatment of non-equilibrium phenomena in thermal plasma flows. J Phys D Appl Phys 41(18):183001. https://doi.org/10.1088/0022$3727 / 41 / 18 / 183001$ 
34. Quintero MC, Rodero A, García MC, Sola A (1997) Determination of the excitation temperature in a nonthermodynamic-equilibrium high-pressure helium microwave plasma torch. Appl Spectrosc 51(6):778-784. https://doi.org/10.1366/0003702971941287

35. Sember V, Gravelle DV, Boulos MI (2002) Spectroscopic study of a supersonic plasma jet generated by an ICP torch with a convergent-divergent nozzle. J Phys D Appl Phys 35:1350-1361

36. Gordon $\mathrm{MH}$, Kruger $\mathrm{CH}$ (1993) Nonequilibrium effects of diluent addition in a recombining argon plasma. Phys Fluids B 5(3):1014-1023. https://doi.org/10.1063/1.860503

37. NIST Atomic Spectra Database (version 5.8), [Online] (2020) National Institute of Standards and Technology. https://www.nist.gov/pml/atomic-spectra-database.

38. Griem HR (1963) Validity of local thermal equilibrium in plasma spectroscopy. Phys Rev 131(3):1170-1176. https://doi.org/10.1103/PhysRev.131.1170

39. Numano M (1990) Criteria for local thermodynamic equilibrium distributions of populations of excited atoms in a plasma. J Quant Spectrosc Radiat Transf 43(4):311-317. https://doi.org/10.1016/00224073(90)90020-7

40. Stehlé C, Hutcheon R (1999) Extensive tabulations of Stark broadened hydrogen line profiles. Astron Astrophys Suppl Ser 140(1):93-97. https://doi.org/10.1051/aas:1999118

41. Wiese WL, Kelleher DE, Paquette DR (1972) Detailed study of the stark broadening of Balmer lines in a high-density plasma. Phys Rev A 6(3):1132-1153. https://doi.org/10.1103/PhysR evA.6.1132

42. Kelleher DE (1981) Stark broadening of visible neutral helium lines in a plasma. J Quant Spectrosc Radiat Transf 25:191-220

43. Balcon N, Aanesland A, Boswell R (2007) Pulsed RF discharges, glow and filamentary mode at atmospheric pressure in argon. Plasma Sources Sci Technol 16(2):217-225. https://doi. org/10.1088/0963-0252/16/2/002

44. Ovsyannikov AA, Zhukov MF (2000) Plasma diagnostics. Cambridge International Science Publishing, Cambridge

45. Zhang H, Wu Y, Sun H, Yang F, Rong M, Jiang F, Wang C, Huang W (2019) Application of calibration-free Boltzmann plot method for composition and pressure measurement in argon free-burning arcs. Plasma Chem Plasma Process 39(6):1429-1447. https://doi.org/10.1007/s11090-019-10018-5

46. Abel NH (1826) Auflösung einer mechanischen Aufgabe. J Reine Angew Math 1826(1):153. https ://doi.org/10.1515/crll.1826.1.153

47. Bockasten K (1961) Transformation of observed radiances into radial distribution of the emission of a plasma. J Opt Soc Am 51(9):943-947. https://doi.org/10.1364/JOSA.51.000943

48. Steenbeck M (1932) Energetik der Gasentladungen. Phys Z 33:809-815

49. Benilov MS, Naidis GV (2010) What is the mathematical meaning of Steenbeck's principle of minimum power in gas discharge physics? J Phys D Appl Phys 43(17):175204. https://doi. org/10.1088/0022-3727/43/17/175204

50. Duan Z, Heberlein J (2002) Arc instabilities in a plasma spray torch. J Therm Spray Technol 11(1):44-51. https://doi.org/10.1361/105996302770348961

51. Ramachandran K, Marqués JL, Vaßen R, Stöver D (2006) Modelling of arc behaviour inside a F4 APS torch. J Phys D Appl Phys 39(15):3323-3331. https://doi.org/10.1088/0022-3727/39/15/015

52. Gindrat M, Dorier JL, Hollenstein C, Refke A, Barbezat G (2004) Characterization of supersonic low pressure plasma jets with electrostatic probes. Plasma Sources Sci Technol 13(3):484-492. https://doi.org/10.1088/0963-0252/13/3/015

53. Gordon S, McBride BJ (1994) Computer program for calculation of complex chemical equilibrium compositions and applications - analysis. NASA-Reference Publication, 1311 part 1. NASA Lewis Research Center, Cleveland, $\mathrm{OH}$

54. Gordon S, McBride BJ (1996) Computer program for calculation of complex chemical equilibrium compositions and applications - user's manual and program description. NASA-Reference Publication 1311, part 2. NASA Lewis Research Center, Cleveland, $\mathrm{OH}$

55. Zhang N, Sun F, Zhu L, Planche MP, Liao H, Dong C, Coddet C (2011) Electron temperature and density of the plasma measured by optical emission spectroscopy in VLPPS conditions. $\mathrm{J}$ Therm Spray Technol 20(6):1321-1327. https://doi.org/10.1007/s11666-011-9681-6

56. de Graaf MJ, Dahiya RP, Jauberteau JL, de Hoog FJ, van de Sande MJF, Schram DC (1990) Thermal plasma source of hydrogen atoms and ions. Colloq Phys 51 (18):C5 387-392

57. Meulenbroeks RFG, Engeln RAH, Beurskens MNA, Paffen RMJ, van de Sanden MCM, van der Mullen JAM, Schram DC (1995) The argon-hydrogen expanding plasma: model and experiments. Plasma Sources Sci Technol 4:74-85

58. de Graaf MJ, Severens R, Dahiya RP, van de Sanden MCM, Schram DC (1993) Anomalous fast recombination in hydrogen plasmas involving rovibrational excitation. Phys Rev E 48(3):2098-2102 
59. Meulenbroeks RFG, van Beek AJ, van Helvoort AJG, van de Sanden MCM, Schram DC (1994) Argon-hydrogen plasma jet investigated by active and passive spectroscopic means. Phys Rev E 49(5):4397-4406

60. van de Sanden MCM, de Regt JM, Schram DC (1993) Recombination of argon in an expanding jet. Phys Rev E 47(4):2792-2797

61. Meeusen GJ, Ershov-Pavlov AA, Meulenbroeks RFG, van den Sanden MCM (1992) Emission spectroscopy on a supersonically expanding argon/silane plasma. J Appl Phys (Melville, NY) 71(9):4156-4163

62. Scoles G (1988) Atomic and molecular beam methods, vol 1. Atomic and molecular beam methods. Oxford University Press, New-York, NY

63. Jongma RT, Rasing T, Meijer G (1995) Two-dimensional imaging of metastable CO molecules. J Chem Phys 102(5):1925-1933. https://doi.org/10.1063/1.468758

64. Mazouffre S, Boogaarts MGH, van der Mullen JAM, Schram DC (2000) Anomalous atomic hydrogen shock pattern in a supersonic plasma jet. Phys Rev Lett 84(12):2622-2625

65. Mazouffre S, Boogaarts MGH, Bakker IS, Vankan P, Engeln R, Schram DC (2001) Transport of ground-state hydrogen atoms in a plasma expansion. Phys Rev E 64(1):016411. https://doi. org/10.1103/PhysRevE.64.016411

66. Ivchenko D, Zhang T, Mariaux G, Vardelle A, Goutier S, Itina TE (2018) On the validity of continuum computational fluid dynamics approach under very low-pressure plasma spray conditions. $\mathrm{J}$ Therm Spray Technol 27(1):3-13. https://doi.org/10.1007/s11666-017-0658-y

Publisher's Note Springer Nature remains neutral with regard to jurisdictional claims in published maps and institutional affiliations. 